\title{
Aversively motivated changes in meal patterns of rats in a closed economy: The effects of shock density
}

\author{
FRED J. HELMSTETTER and MICHAEL S. FANSELOW \\ Dartmouth College, Hanover, New Hampshire
}

\begin{abstract}
Rats lived continuously in an operant chamber in which they were able to press a bar to obtain food on a chained FR50:CRF schedule that allowed them control of both the size and frequency of individual meals. Independent groups of animals were scheduled to receive 12, 24, 48, or 96 electric shocks per day, which were given randomly in time and independent of the subjects' behavior. Rats could avoid shock by remaining in a safe area of the chamber, but they were always at risk while barpressing. The introduction of shock resulted in a number of changes in feeding patterns. In rats exposed to a possible 12 shocks/day, meal size increased whereas meal frequency changed very little. At 24 shocks/day, meal frequency decreased whereas meal size increased such that net intake remained stable relative to a preshock baseline period. As shock density was increased to 48 or 96 shocks/day, total intake was suppressed. At 96 shocks/day, both meal frequency and meal size decreased dramatically. Shock-related changes were also observed in rates of operant responding and in the amount of time the animals engaged in feeding-related behavior. All of the animals were able to achieve a greater than $50 \%$ reduction in the total number of shocks received relative to equivalent random samples of their position in the apparatus taken during baseline. These results support the position that the nature of defensive changes in feeding behavior that are seen when an aversive stimulus is introduced into a simulated foraging situation varies as a function of risk.
\end{abstract}

Optimal foraging theory predicts that animals will tend to maximize caloric intake relative to the costs involved in obtaining food. It is assumed that natural selection operates on the mechanisms of consumption such that strategies leading to more efficient use of time and energy will be favored (Krebs \& Davies, 1981). Although the expense in time and energy required to procure and consume food constitutes one aspect of the cost involved in foraging, there are likely to be other costs associated with this activity in the wild. Since it is reasonable to assume that an animal might often be required to leave an area of relative safety in order to gain access to a food source, foraging may also involve an increased risk of predation (Krebs, 1980). We have recently begun to study the effects of predatory risk on foraging behavior in the laboratory (Fanselow \& Lester, 1988; Fanselow, Lester, \& Helmstetter, 1988).

Collier and his associates (e.g., Collier, 1983) have developed a laboratory model of foraging and consummatory behavior that may reflect a number of critical features that animals are likely to encounter in their natural environment. Importantly, these features have been absent in traditional experimental designs used to study food-

This research was supported by NIMH Grant MH39786 to M.S.F., who is now in the Department of Psychology at UCLA. Requests for reprints should be sent to F. J. Helmstetter, Department of Psychology, Garland Hall, P.O. Box 413, University of Wisconsin-Milwaukee, Milwaukee, WI 53201. reinforced operants. In Collier's experiments, animals live in an operant chamber for extended periods during which they gain access to the sole source of food by performing a response such as barpressing. The costs associated with foraging are modeled by chained schedules of reinforcement. The first component of the schedule is referred to as the procurement cost and corresponds to the work required for an animal to find and obtain food. The terminal component is referred to as consumption and corresponds to work related to handling, preparing, and consuming the food item. In a typical experiment, completion of the procurement component of the schedule provides access to the consumption phase or, "meal." Once an animal has initiated a meal, it will continue to obtain food until it fails to respond for some predetermined period of time. When this occurs, the animal must complete another procurement requirement to take another meal. Thus, in this situation, the animal has control over both the frequency and size of feeding bouts. This situation is normally described as a "closed economy," since the animal's total nutritional requirements may be satisfied through its interaction with the contingencies of interest, and the subject is provided with no supplemental food outside of the experimental situation (Hursh, 1980).

A variety of species display characteristic patterns of plasticity in feeding behavior in response to manipulation of the cost of food in a closed economy. In Collier's experiments, as the cost of procurement (access to a food source) increased, animals tended to initiate fewer meals 
but showed a compensatory increase in meal size such that total intake and body weight tended to remain stable (Collier, Hirsch, \& Hamlin, 1972). When the number of responses required to obtain each unit of food in the consumption component is increased, however, animals normally respond by increasing their rate of responding. This response to consumption cost is seen regardless of the current procurement cost. Thus, the behavioral effects of changing the cost of procurement and consumption are different and seem to be independent (Collier, 1983).

When unsignaled electric shock is introduced into a model foraging situation, similar to that described above, in which animals may avoid shock by remaining in a safe area but must risk exposure to shock in order to obtain food, they respond with a decrease in meal frequency and increase in meal size similar to that seen when the work required to complete a procurement component is increased (Fanselow \& Lester, 1988; Fanselow et al., 1988). We have demonstrated that the changes in behavior produced by this procedure are accompanied by a reduction in the amount of shock an animal receives and thus may be considered a form of shock avoidance. These changes in meal pattern are specific consequences of shock associated with the foraging situation (Fanselow, et al., 1988). The present experiment represents a more detailed parametric analysis, in terms of shock frequency, of the changes in meal patterns and shock avoidance in this preparation.

\section{METHOD}

\section{Subjects}

A total of 16 female Long-Evans rats served as subjects. The animals were bred locally from stock obtained from Blue Spruce Farms (Altamont, NY) and until the beginning of the experiment were maintained 2 per cage on ad-lib high-protein lab chow (Agway) and water. The subjects weighed between $220-290 \mathrm{~g}$ and were $110-130$ days old at the start of the experiment. A 14:10-h light:dark cycle was in effect until the beginning of the experiment. During the experiment, a 12:12-h light:dark cycle was in effect with lights out at $2000 \mathrm{~h}$.

\section{Apparatus}

Animals lived throughout the study in one of eight modified operant chambers (see Fanselow \& Lester, 1988, for a drawing of the apparatus). The chambers were constructed of clear acrylic plastic and measured $58.5 \times 26 \times 20.6 \mathrm{~cm}$. Each chamber was divided into two compartments by a plastic hurdle that was $5.75 \mathrm{~cm}$ high. The small compartment, or nest area, measured $16 \times 25 \mathrm{~cm}$ and was filled with $500 \mathrm{ml}$ of sawdust. A steel response lever was mounted approximately $3 \mathrm{~cm}$ from the floor on the wall opposite the nest area. The lever measured $5 \mathrm{~cm}$ wide and $1.5 \mathrm{~cm}$ thick, and protruded $1.5 \mathrm{~cm}$ into the chamber. A round cue light $(1 \mathrm{~cm}$ in diameter) was mounted on the wall $9 \mathrm{~cm}$ above the response lever, and a food hopper was placed $9 \mathrm{~cm}$ to the left of the lever. The food hopper was connected to an automated pellet dispenser though which 45-mg food pellets (Noyes formula A) could be delivered. A water spout was located $9 \mathrm{~cm}$ to the left of the response lever. The floor of the large compartment, or foraging area, was composed of 25 brass rods ( $3 \mathrm{~mm}$ in diameter, spaced $1.5 \mathrm{~cm}$ center to center) through which shock could be delivered. Shock was generated with a high voltage source wired in series with a chain of neon bulbs attached to the grid floor. Shock intensity was initially set at $1 \mathrm{~mA}$ and was monitored throughout the experiment. Shock could be delivered to the floor of the large compartment only. The nest area was always shock-free.

The apparatus was controlled by a dedicated microcomputer that recorded all barpress responses, monitored a pressure activated switch that remained closed when an animal was in the nest area, and operated the pellet dispensers, cue lights, and shock generators.

\section{Procedure}

Four independent groups of animals $(n=4)$ were assigned to receive shock densities of $12,24,48$, or 96 shocks per day. Each day the computer generated a series of shock times that were randomly distributed over a $24-\mathrm{h}$ period. At each of these designated times, the position of the animal (in the nest area or not) was determined by reading the state of a pressure-activated switch mounted under the nest area. The procedure during the shock phase differed only in that a 1-mA, 1-sec shock was administered $25 \mathrm{msec}$ after recording the animal's position. Thus, during both baseline and shock periods, the rat's location was randomly sampled at the shock density programmed for that group. The minimum intershock interval was $1 \mathrm{sec}$.

On Day 1, the animals were removed from their home cages, placed in one of the experimental chambers, and given the opportunity to barpress for food on a chained FRI :continuous reinforcement (CRF) schedule. Activation of the cue light signaled entry into the CRF component. The subjects were not deprived prior to the beginning of the experiment and no shaping procedures were used. If a rat failed to acquire the response reliably within 2 days, it was eliminated from the experiment and replaced with an individual of similar weight, sex, and strain. Two animals were rejected in this manner. When all of the subjects evidenced stable responding at FR1, the fixed ratio component (procurement cost) was gradually increased to FR50 over a period of 12 days. All animals remained on an FR50:CRF schedule throughout the remaining two phases of the experiment. The subjects were free to initiate the sequence at any time. If an animal ceased barpressing for 4 min while eating or after having partially completed a procurement requirement, the meal was terminated or the procurement counter was reset. The maximum amount of time an animal could spend in procurement was therefore $196 \mathrm{~min}$. This constraint was imposed so that variability in average response rates would be reduced. An animal could remain in the CRF component indefinitely provided that no more than 4 min elapsed between responses.

The first phase consisted of a 7-day baseline period during which the subjects' meal patterns and the number of instances in which they would have received shock if the shock apparatus had been operative were recorded. The computer generated shock times at the density programmed for each group, as described above. At each of these times, the position of the animal relative to the safe area of the chamber was recorded. Following this baseline period, the shock source was enabled. The shock phase continued for 7 days.

Each day at approximately 17:30 throughout the experiment, the experimenter weighed each animal, replaced the sawdust bedding in the nest area, and cleaned the drop trays. At this time, the presence of food pellets in the drop tray and nest area was recorded.

\section{RESULTS}

The following variables were analyzed for each animal for each day during the baseline and shock periods: (1) body weight, (2) number of meals completed, (3) whether each meal was initiated in the light or dark portion of the day/night cycle, (4) average meal size, (5) total number of pellets consumed, (6) average response rate during the consumption component of each meal, (7) average time 
spent in the consumption component of each meal, (8) average time spent in the procurement component of each meal, (9) total number of shocks (or equivalent samples during baseline) received or avoided as determined by the animal's position in the apparatus, (10) number of shocks received during the light versus the dark portions of the light-dark cycle, and (11) number of shocks received during the performance of procurement and consumption components of meals. If an animal failed to initiate any meals on a given day, it was given a score of zero for average meal size for that day.

Each feeding-related variable was subjected to a threeway analysis of variance (ANOVA) that treated group (shock density) as a between-subject treatment and phase (baseline or shock) and day (1-7) as repeated measures. The means for each shock-density group for each phase of the experiment (collapsed over days) may be found in Table 1 along with $p$ values summarizing the results of the overall ANOVA. Mean values of shock-related variables for each group may be found in Table 2. Independent groups of rats were scheduled to receive different densities of shock; therefore, the position of the animals was sampled at different frequencies for each group during both phases of the experiment. Consequently, the absolute frequency of shock receipt would likely differ between groups regardless of the subjects' behavior. Prior to an ANOVA, shock frequency values for each day during the shock phase were converted to a percentage of mean baseline values (the equivalent nonshock baseline samples averaged across days). Data for the five shockrelated variables (presented as absolute frequencies) in Table 2 were transformed in this manner and subjected to a 4 (group) $\times 7$ (day) repeated measures ANOVA. A summary of this analysis, represented by $p$ values for group and day main effects as well as for the group $x$ day interaction, may also be found in Table 2 . For consistency and clarity in presentation, the data in Figures 1 through 7 are presented as percentages of mean baseline values.

Inspection of Figure 1 indicates that rats responded to unsignaled footshock in this preparation with changes in both meal frequency and meal size, a finding that has been reported previously (Fanselow et al., 1988). Interestingly, the magnitude and direction of this effect seemed to be related to the density of programmed shock. In the 12 shocks/ day group, meal frequency remained close to baseline values. Rats exposed to a possible 24 shocks per day showed small reductions in meal frequency, which remained relatively stable over the 7-day shock period, whereas animals exposed to high shock densities showed a large initial decrease in frequency, which partially recovered in the 48 shocks/day group but did not do so in the 96 shocks/day group. Groups 12 and 24 showed moderate and stable increases in average meal size. Although meal size initially dropped to $50 \%$ of preshock baseline in Group 48 on Day 1 of the shock phase, it eventually showed a large increase

Table 1

Feeding-Related Variables Collapsed Over Baseline and Shock Phases

\begin{tabular}{|c|c|c|c|c|c|c|c|c|c|c|c|c|c|c|c|}
\hline \multirow[b]{3}{*}{ Variable } & \multicolumn{8}{|c|}{ Shock Density } & & & & & & & \\
\hline & \multicolumn{2}{|c|}{12} & \multicolumn{2}{|c|}{24} & \multicolumn{2}{|c|}{48} & \multicolumn{2}{|c|}{96} & \multicolumn{7}{|c|}{ ANOVA } \\
\hline & Baseline & Shock & Baseline & Shock & Baseline & Shock & Baseline & Shock & Group & Phase & $\mathbf{G} \times \mathbf{P}$ & Day & $G \times D$ & $\mathrm{P} \times \mathrm{D}$ & $G \times P \times D$ \\
\hline Body weight (grams) & 231.46 & 242.00 & 252.07 & 275.11 & 272.43 & 272.11 & 268.82 & 251.14 & 0.206 & 0.086 & 0.000 & 0.084 & 0.054 & 0.257 & 0.005 \\
\hline Total daily intake (grams) & 16.93 & 19.29 & 18.41 & 21.13 & 23.13 & 17.42 & 19.27 & 6.10 & 0.001 & 0.000 & 0.000 & 0.002 & 0.084 & 0.042 & 0.038 \\
\hline $\begin{array}{l}\text { Meals per day } \\
\text { Average meal size }\end{array}$ & 7.00 & 6.82 & 6.00 & 5.21 & 8.89 & 4.71 & 8.29 & 2.18 & 0.015 & 0.000 & 0.002 & 0.085 & 0.040 & 0.493 & 0.530 \\
\hline (number of pellets) & 53.42 & 68.37 & 70.09 & 97.19 & 58.79 & 80.29 & 55.21 & 44.79 & 0.036 & 0.020 & 0.088 & 0.022 & 0.047 & 0.023 & 0.051 \\
\hline $\begin{array}{l}\text { Average consumption time } \\
\text { (min/meal) }\end{array}$ & 12.13 & 16.79 & 18.94 & 24.07 & 13.54 & 18.76 & 12.36 & 16.23 & 0.066 & 0.052 & 0.313 & 0.061 & 0.081 & 0.012 & 0.360 \\
\hline $\begin{array}{l}\text { Average consumption response } \\
\text { rate (response/sec) }\end{array}$ & 0.08 & 0.09 & 0.07 & 0.07 & 0.08 & 0.08 & 0.09 & 0.07 & 0.284 & 0.245 & 0.080 & 0.656 & 0.454 & 0.161 & 0.029 \\
\hline $\begin{array}{l}\text { Average procurement time } \\
(\text { min/meal) }\end{array}$ & 3.16 & 2.90 & 3.60 & 3.30 & 2.18 & 1.81 & 2.95 & 3.38 & 0.434 & 0.118 & 0.595 & 0.146 & 0.332 & 0.582 & 0.236 \\
\hline Meals in light & 1.82 & 1.39 & 2.50 & 1.14 & 2.29 & 0.46 & 3.14 & 0.54 & 0.495 & 0.000 & 0.004 & 0.001 & 0.825 & 0.051 & 0.633 \\
\hline Meals in dark & 5.18 & 5.43 & 3.50 & 4.07 & 6.61 & 4.25 & 5.14 & 1.64 & 0.002 & 0.010 & 0.011 & 0.048 & 0.007 & 0.019 & 0.661 \\
\hline
\end{tabular}

Table 2

Shock-Related Variables Collapsed Over Baseline and Shock Phases

\begin{tabular}{|c|c|c|c|c|c|c|c|c|c|c|c|}
\hline \multirow[b]{3}{*}{ Variable } & \multicolumn{8}{|c|}{ Shock Density } & & & \\
\hline & \multicolumn{2}{|c|}{12} & \multicolumn{2}{|c|}{24} & \multicolumn{2}{|c|}{48} & \multicolumn{2}{|c|}{96} & \multicolumn{3}{|c|}{ ANOVA } \\
\hline & Baseline & Shock & Baseline & Shock & Baseline & Shock & Baseline & Shock & Group & Day & $G \times D$ \\
\hline Total shocks received & 3.82 & 1.61 & 7.50 & 3.36 & 12.46 & 2.00 & 20.11 & 8.29 & 0.510 & 0.007 & 0.960 \\
\hline Shocks in light & 1.82 & 0.32 & 4.54 & 1.14 & 5.04 & 0.36 & 9.32 & 3.32 & 0.429 & 0.189 & 0.717 \\
\hline Shocks in dark & 2.00 & 1.29 & 2.96 & 2.21 & 7.43 & 1.64 & 10.79 & 4.96 & 0.157 & 0.000 & 0.999 \\
\hline Shocks during procurement & 0.32 & 0.07 & 0.07 & 0.00 & 0.50 & 0.00 & 1.54 & 0.00 & 0.426 & 0.432 & 0.470 \\
\hline Shocks during consumption & 0.82 & 0.21 & 1.64 & 0.71 & 2.21 & 0.07 & 5.64 & 0.00 & 0.012 & 0.550 & 0.131 \\
\hline
\end{tabular}



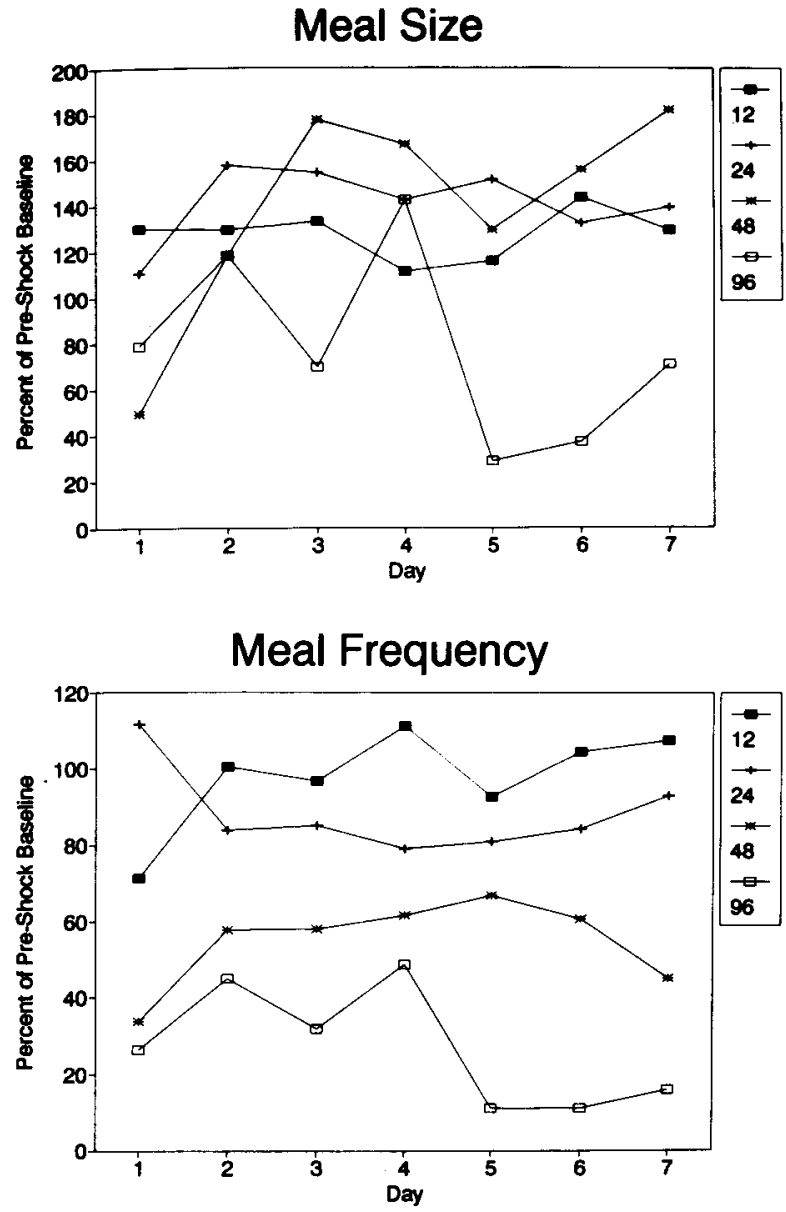

Figure 1. The average meal size and average meal frequency of rats receiving 12, 24, 48, or 96 shocks per day during the 7-day shock phase expressed as a percentage of mean baseline values calculated for each animal from a comparable 7-day baseline period prior to shock.

and remained stable. At the highest shock density, meal size decreased on 5 of the 7 days.

The data plotted in Figure 2 indicate that the relative reduction in meal frequency differed for meals taken during both portions of the light-dark cycle. The number of meals completed during the lights-on period (averaged over the shock phase), and thus the percentage of baseline, was reduced for all groups. Furthermore, this reduction appeared to be directly related to the number of programmed shocks. For meals completed during the lights-off period, Groups 12 and 24 actually showed a slight increase relative to baseline values, although overall meal frequency (light + dark) decreased. Groups 48 and 96 showed a reduction in the number of meals during the dark period, but this relative reduction was smaller than that seen during the light period in the same subjects. Thus, although animals decreased overall meal frequency as shock density increased, meals initiated during light versus dark periods were differentially affected.
Figure 3 (upper panel) indicates that for Groups 12 and 24 , body weight increased slightly relative to the baseline period and remained stable throughout the shock phase. Group 48 showed an initial loss in body weight, which gradually recovered to baseline levels, whereas Group 96 continued to lose weight throughout the shock phase. A somewhat comparable pattern may be seen in total food intake, depicted in the lower panel of Figure 3. Daily total intake increased slightly and remained stable for the two low density groups, whereas Group 48 showed an initial decrease, which partially recovered. A similar initial decrease seen in Group $\mathbf{9 6}$ was followed at first by a slight increase and then by a substantial decrease in the amount of food consumed late in the shock phase.

The average amount of time spent in the consumption component of each meal and the average response rate during this component are shown in Figure 4. Consumption time tended to increase for each group over the 7-day shock phase, although Groups 48 and 96 showed an initial decrease following activation of the shock source. Consumption rate showed less variability over the shock phase. Groups 24 and 48 responded at a rate very similar to their baseline throughout the shock phase. Group 96 showed a small but consistent decrease in rate and Group 12 increased on Days 4 and 5 . The time spent performing the procurement component of each meal did not differ between groups as a function of either within-subject variable. Since all of the subjects were on the same FR50:CRF schedule during both phases of the study, procurement rate was the inverse of procurement time and was not analyzed.

During the baseline period, the position of each animal relative to the shock grid was recorded at a series of randomly determined times at a rate corresponding to its programmed shock density. On the average, the rats tended

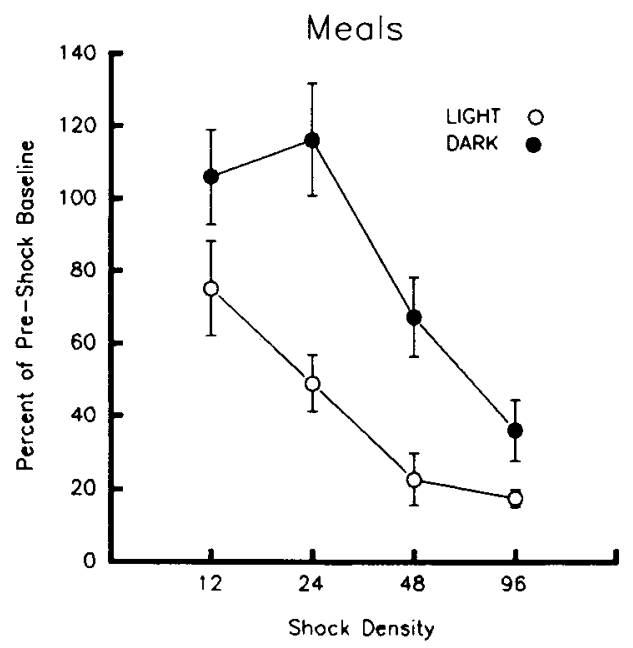

Figure 2. Percentage of baseline meal frequency (collapsed over the shock period) during 12-h periods of light versus dark during the shock phase. All groups showed a larger reduction in meals taken during the light portion of the day-night cycle. 


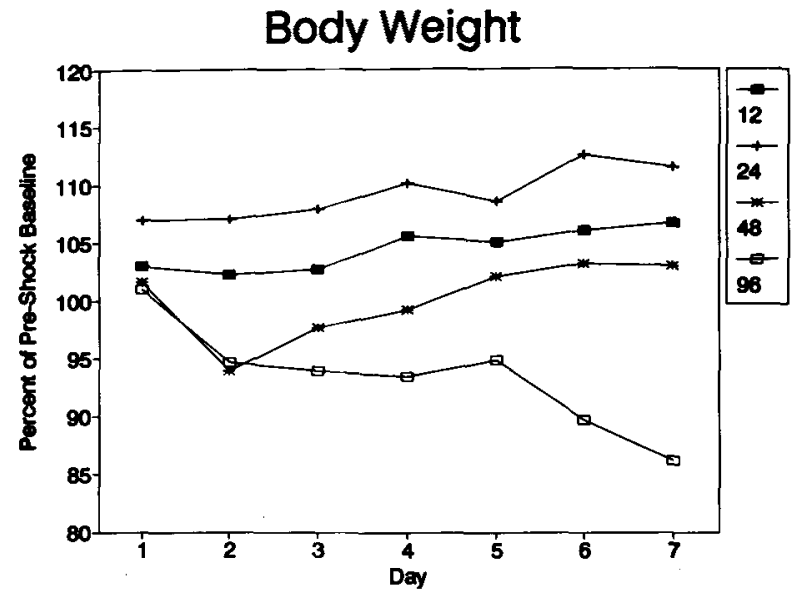

Total Intake

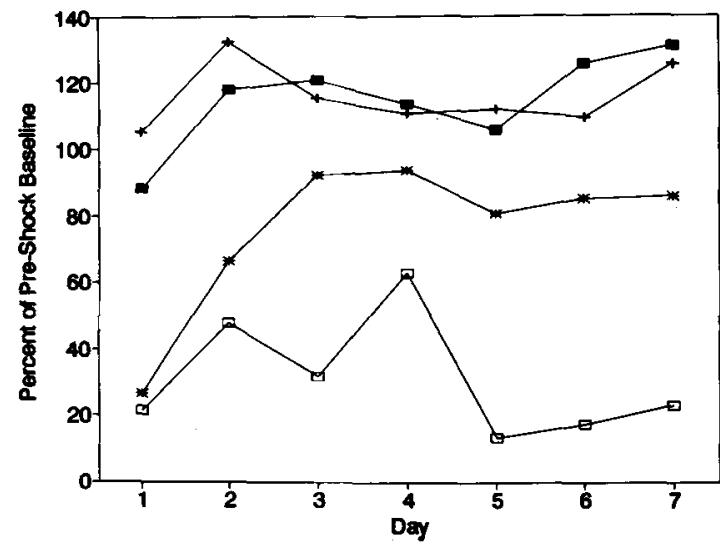

Figure 3. Body weight and total food intake expressed as a percentage of the mean preshock baseline for each day during the shock phase.

to be present on the grid floor for $27.75 \%$ of baseline samples, whereas during the shock period, animals received on average only $9.9 \%$ of programmed shocks (see Table 2 ). The top panel of Figure 5 indicates that over the course of the shock phase, all groups were able to avoid shock by reducing the amount of time spent on the grid floor relative to the average number of equivalent samples taken during baseline. This reduction in shock receipt was relatively small on the first day of the shock phase, but the daily number of shocks received dropped to less than $50 \%$ of baseline for all groups by the end of the experiment. The 48 shocks/day group was most effective in avoiding shocks by consistently being absent from the grid floor for nearly all shock samples during the last 4 days of the shock phase.

The lower panel of Figure 5 plots the "ingestion-related exposure" [meal frequency $\times$ (average consumption time + average procurement time)] for each day during the shock phase. Thus, one may compare the percentage of shock received or avoided (shown in Figure 5) with the percentage of time spent engaged in feeding-related activity for each group. Interestingly, the low shock density groups (12 and 24 shocks/day) increased, or did not change, the average amount of time spent on the grid floor obtaining and consuming food, whereas the two remaining groups showed a large initial decrease. As was the case for both meal frequency and total intake, Group 48 showed a gradual recovery toward baseline levels, whereas Group 96 remained relatively suppressed throughout this phase of the experiment.

The average number of shocks received during the performance of procurement and consumption meal components is given in Figure 6. The animals exposed to the lowest shock density reduced the number of procurementrelated shocks by nearly $80 \%$. The remaining groups were able to completely avoid shock during performance of the procurement requirement. All groups reduced consumptionrelated shocks, although the size of this reduction varied. Groups 12,48 , and 96 showed reductions in consumptionrelated shock receipt that were very similar to the reduc-
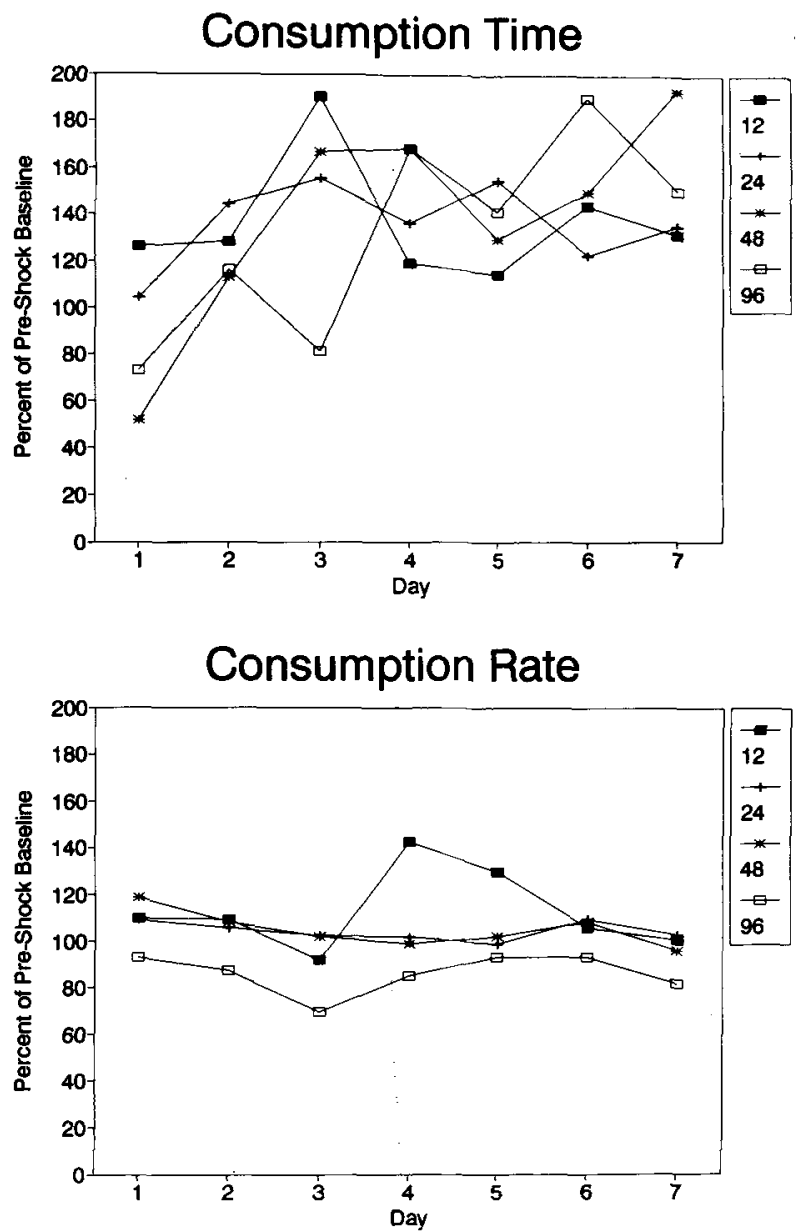

Figure 4. The average amount of time spent engaged in food consumption and the average response rate for the consumption (on a continuous reinforcement schedule) component of meals on each day of the shock phase relative to preshock baseline values. 


\section{Total Shock Received}

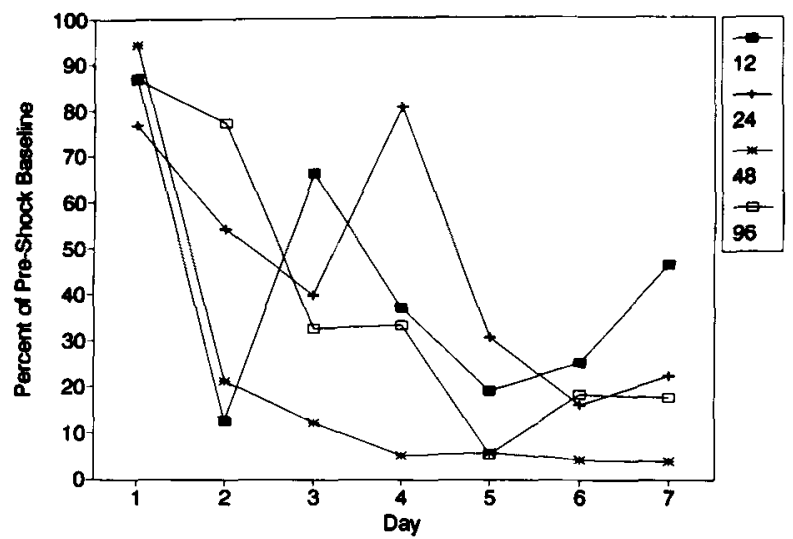

Ingestion-Related Exposure

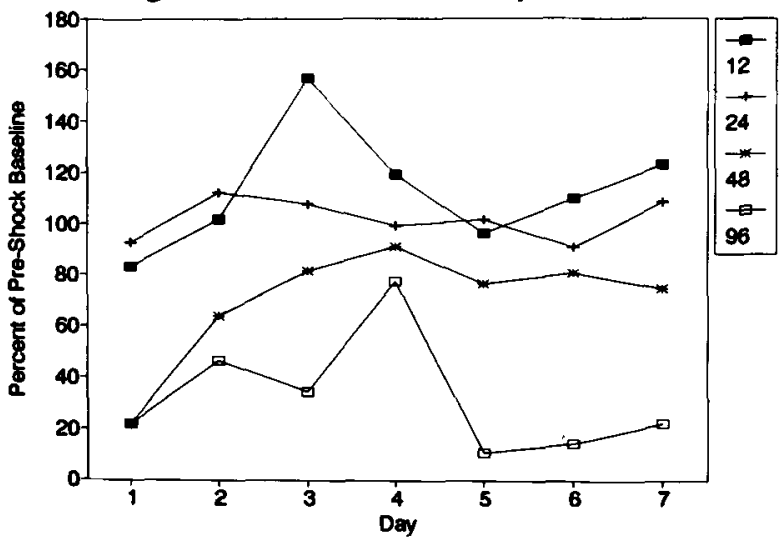

Figure 5. The number of footshocks received during the second phase of the experiment. Shock receipt or avoidance is inferred from the animal's position in the apparatus (nest box vs. grid floor) at the time of shock delivery. Data are expressed as a percentage of equivalently programmed position samples recorded during the baseline period.

tion in procurement-related shock receipt for each group. Group 24, however, showed only a $50 \%$ reduction in consumption-related shocks even though procurement-related shocks were eliminated.

The pattern of relative shock avoidance also seemed to differ with respect to shocks occurring during the light versus dark portions of the light-dark cycle. Figure 7 indicates that all of the subjects achieved rather large reductions in average shock receipt during the light hours. Three out of the four groups showed similar, albeit smaller, reductions in average shock receipt during the dark portion of the cycle. The 24 shocks/day group failed to decrease shock receipt at night, although the performance of this group during the day was similar to that of the others.

\section{DISCUSSION}

If the risk of encountering electric shock represents a form of cost imposed on foraging in the closed economy, then we would predict that the behavioral effects of shock, or of the risk of receiving shock, would to some extent resemble the effects of manipulating other forms of cost in this paradigm. This was seen to be the case. The results of this experiment confirm that when moderately high frequency unpredictable shock is introduced into a simulated foraging situation, rats respond with adjustments in meal size and/or frequency. Although the change in meal frequency in Group 24 was smaller than we would have expected on the basis of previous experience with these parameters (e.g., Fanselow et al., 1988), it may have been caused by the relatively low meal frequency during baseline in this group. Nonetheless, when absolute meal frequency during the shock phase is considered, meal frequency is seen to have a strong inverse relationship to the density of programmed shocks (see Table 1).

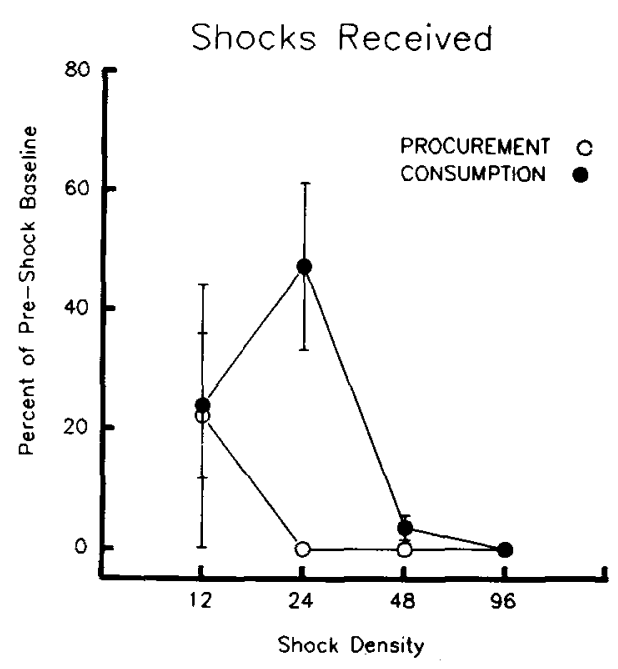

Figure 6. Shock receipt as a function of meal component and shock density. Means have been collapsed over days, as in Figure 2.

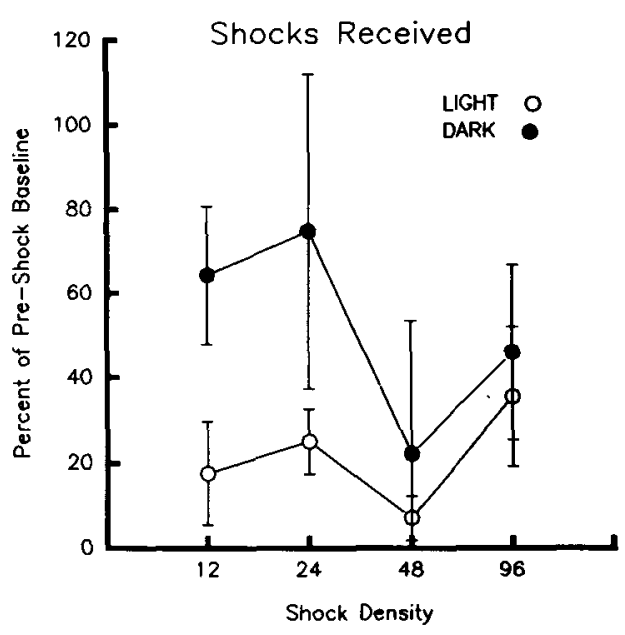

Figure 7. Shock receipt as a function of photoperiod and shock density. The net reduction in shock receipt for samples taken during the light portion of the cycle was greater for all groups. 
For all groups, introduction of shock resulted in changes in behavior such that the number of shocks received was reduced relative to an equivalent number of random samples of the animals' position recorded during the preshock baseline period. This reduction was greater than $50 \%$ in all groups by the end of the shock phase and approached $95 \%$ in the 48 shocks/day group. It is clear that rats are able to avoid shock in this situation when they are given a safe area in the chamber. Table 2 indicates that although animals spent most of their time in the nest box during the baseline period, the introduction of shock caused them to spend even less time on the grid floor. Since shocks occurring during performance of barpressing were only a small fraction of the total number (see Table 2), most of the shocks were avoided by the animals spending increased amounts of "free" time in the nest box.

Even though a relatively small number of total shock samples occurred while animals were engaged in feedingrelated behavior, all groups showed a greater than $50 \%$ reduction in the number of shocks received during the performance of procurement or consumption responses relative to position samples taken during baseline. It would appear that this reduction in shock receipt is at least in part due to the density-related change in meal frequency found in some groups. By reducing absolute meal frequency, the rats exposed to high shock densities (48 or 96 shocks/day) reduced meal-related time spent at risk (Figure 5). However, at low shock densities, small changes in meal frequency, coupled with an increase in meal size and little or no change in consumption or procurement rates, resulted in a slight net increase in the minimum amount of time at risk of receiving shock. Nonetheless, these animals in the 12 and 24 shocks/day groups were able to avoid over $50 \%$ of the shocks occurring during meals. This seems to have been achieved, in part, through a reorganization of meal patterns with respect to the light-dark cycle (see Figures 2 and 7). All of the subjects tended to take more meals in the dark during the baseline phase, and this preference for nocturnal feeding was increased after shock was introduced (Table 1). Since shock times were randomly generated and thus uniformly distributed over each 24- $h$ period, the animals should not have reduced the effective probability of being shocked by redistributing exposure into a particular subset of that period (i.e., at night). It is therefore possible that the apparent relationship between this change in feeding pattern and shock receipt is an artifact considering the relatively low sampling rate for shocks ( 0.5 or 1.0 each hour) in these two groups.

Surprisingly, the animals did not respond to the risk of receiving shock by increasing their rate of barpressing. The amount of time used to perform the procurement component (and therefore the procurement rate) did not change significantly relative to baseline meal data. The lack of a large and consistent increase in consumption rate necessarily resulted in longer meals when meal size increased. This increase in consumption time offset the savings in minimum time at risk that would have been achieved in the low density groups on the basis of meal frequency reductions alone. The failure to adjust response rates is particularly interesting in light of the fact that rats required to leave a warm nest box to forage in a cold environment decrease meal frequency but defend total intake by eating faster (Johnson \& Cabanac, 1982). It is possible that a greater degree of plasticity in consumption rates would be seen if shock were introduced with a higher consumption cost in place (e.g., Collier, 1983), since in the present situation, our informal observations indicate that the animals' consumption response rate is partially limited by the rate at which it can handle and consume each pellet. Further experiments will be needed to see if this lack of rate adjustment is peculiar to the CRF consumption schedule used here.

Despite the fact that the average amount of time spent in the consumption component of individual meals increased during the shock phase, the average number of shocks per day that the animals received while feeding was dramatically reduced. This point is well illustrated by the 96 shocks/day group, which increased its average consumption time by $30 \%$ while completely avoiding shocks during feeding (Tables 1 and 2). It should be noted that even though average consumption time appears to increase, the average absolute period of exposure required for consumption (average consumption time $\times$ meal frequency) was consistently reduced. For example, the rats in the 96 shocks/day group spent an average of $102.46 \mathrm{~min} /$ day barpressing in the CRF component during the baseline phase and only $35.38 \mathrm{~min} /$ day feeding after shock was introduced. Even though exposure to shock was reduced by the animals' taking fewer meals, other factors could have contributed to the dramatic reduction in shock receipt during meal periods. Since the consumption component of the schedule remained in effect as long as the subjects' interresponse interval was less than $4 \mathrm{~min}$, some rats might have learned to avoid shock by remaining in the nest box during meals, spending only enough time on the grid floor to perform the response and retrieve a pellet. Future studies in which the animals' behavior is videotaped or additional random samples of the animals' position in the apparatus are taken will be needed to determine if rats use such behavioral "strategies" in this situation.

Even though shock was programmed to occur randomly throughout each 24-h period, the net change in meal frequency differed in the light and dark phases (Figure 2). Each group of rats showed a larger relative change in meal frequency during the light phase. Following the introduction of shock, the 12 and 24 shocks/day groups increased, whereas the remaining groups decreased, the number of meals taken during the dark phase. Averaged across the four groups, under baseline conditions, the rats completed approximately $67 \%$ of all meals during the dark phase. When shock was introduced, this tendency toward nocturnal feeding increased, with the subjects now initiating approximately $80 \%$ of all meals in the dark phase. 
The apparent difference in plasticity of meal patterns in the light versus in the dark phases raises a number of important questions about the possible status of the meal pattern changes observed in this preparation as a speciestypical defensive response to the threat of predation. The rat may have developed its strong nocturnal foraging tendency in response to pressure from visually guided predators. This putative defensive function of nocturnal feeding may have resulted in the bias toward greater net suppression of feeding during the light phase that was observed here. Several species show differential plasticity in species-typical defensive behavior in the light versus in the dark (e.g., Rovee-Collier, Capatides, Fagen, \& Negri, 1983) and the apparent resistance to suppression of feeding in the dark versus in the light may represent a form of biological constraint on the performance of the rat (LoLordo, 1979).

However, a recent study from our laboratory designed to address this question more directly failed to support the existence of dark-light asymmetries in meal pattern changes in this preparation. Lester and Fanselow (1992) argued that if nocturnal feeding has evolved as a defensive response for the rat, and foraging during daylight hours has historically resulted in an increased risk of predation, then subjects should have a biological predisposition to associate the light phase of the experiment with aversive stimulation. When unsignaled shock was selectively programmed to occur only in the light or dark phase, rats were able to successfully discriminate and reduce consumption (and presumably the number of shocks received) during the "dangerous" phase with minimal disruption in consummatory behavior during the "safe" phase. However, the basic pattern of effects and the magnitude of changes in consummatory behavior did not differ as a function of the photoperiod in which shock was presented; therefore, the data do not support "selective associations" between light and shock. In contrast to the present study, when the same 15 shocks/day were distributed evenly over the entire 24-h period, the relative proportion of meals taken in the dark phase did not change following the introduction of shock (75\% baseline vs. $74 \%$ shock).

Although there are several potential reasons for the lack of selective shifts in meal patterns as a function of photoperiod similar to those observed here, the most likely explanation may be related to the fact that Lester and Fanselow (1992) used a chained FR1:CRF schedule of reinforcement. Our previous work has shown that the effects of procurement cost as modeled by the FR component of the chained schedule interact with the effects of unsignaled shock (Fanselow et al., 1988). Increasing procurement cost and increasing shock density can both result in a decrease in meal frequency. Since Lester and Fanselow (1992) used a relatively low shock density (15 shocks/day) and a very small procurement cost (FR1), the combination of these factors may have resulted in differences between their data and the present results. Additional research will be needed to determine the extent to which feeding patterns across the light-dark cycle interact with the behavioral costs of shock.
Fanselow and Lester (1988) predicted changes in topography of the rat's defensive behavior as a function of the risk of predation. Although our recent work supports this notion and discusses shock induced changes in meal patterns in the general context of the theory (Fanselow et al., 1988), the present study represents a parametric manipulation of risk as modeled by density of electric shock in a foraging situation. In support of predatory imminence theory, we find that as the risk of shock is increased, the changes in behavior that serve to avoid shock and defend food consumption seem to take different forms. If one views the density of aversive stimulation as a continuous variable that is an important factor in selecting the topography of defensive behaviors, the reorganization of meal patterns seen at low to moderate density levels $(0.5-2$ shocks $/ \mathrm{h})$ may give way to other responses in the animals defensive repertoire (such as freezing) when shock is presented 3-4 times/h (Bolles \& Riley, 1973). Low shock densities evoke subtle changes in meal patterns, with intake remaining stable or increasing, whereas high shock densities result in dramatic changes in meal patterns, a reduction in net consumption, and response-suppressing effects similar to those observed in other aversive conditioning situations.

\section{REFERENCES}

Bolles, R. C., \& Riley, A. L. (1973). Freezing as an avoidance response: Another look at the operant-respondent distinction. Learning \& Motivation, 4, 268-275.

Collier, G. H. (1983). Life in a closed economy: The ecology of learning and motivation. In M. D. Zeiler \& P. Harzem (Eds.), Advances in analysis of behavior: Biological factors in learning (pp. 223-274). Chichester, U.K.: Wiley.

Collier, G. H., Hirsch, E., \& Hamuin, P. (1972). The ecological determinants of reinforcement in the rat. Physiology \& Behavior, 9, 705-716

FANSElow, M. S., \& Lester, L. S. (1988). A functional behavioristic approach to aversively motivated behavior: Predatory imminence as a determinant of the topography of defensive behavior. In R. C. Bolles \& M. D. Beecher (Eds.), Evolution \& learning (pp. 185-211). Hillsdale, NJ: Erlbaum.

Fanselow, M. S., Lester, L. S., \& Helmstetter, F. J. (1988). Changes in feeding and foraging as an antipredator defensive strategy: A laboratory simulation using aversive stimulation in a closed economy. Journal of the Experimental Analysis of Behavior, 50, 361-374.

HURSH, S. J. (1980). Economic concepts for the analysis of behavior. Journal of the Experimental Analysis of Behavior, 34, 435-452.

Johnson, K. G., \& CABANaC, M. (1982). Homeostatic competition between food intake and temperature regulation in rats. Physiology \& Behavior, 28, 675-679.

KREBS, J. R. (1980). Optimal foraging, predation risk and territory defence. Ardea, 68, 83-90.

KREBS, J. R., \& DAvies, N. B. (EDS.) (1981). Behavioral ecology. Sunderland, MA: Sinauer.

LeSTER, L. S., \& FANSELOW, M. S. (1992). Nocturnality as a defensive behavior in the rat: An analysis in terms of selective association between light and aversive stimulation. Psychological Record, 42, 221-253. LoLoRDo, V. M. (1979). Selective associations. In A. Dickinson \& R. A. Boakes (Eds.), Mechanisms of learming and motivation (pp. 367398). Hillsdale, NJ: Erlbaum.

Rovee-Collier, C., Capatides, J. B., Fagen, J. W., \& Negri, V. (1983). Selective habituation of defensive behavior: Evidence for predator-prey synchrony. Animal Learning \& Behavior, 11, 127-133.

(Manuscript received August 6, 1989; revision accepted for publication September 26, 1992.) 